\title{
Dor lombar em mulheres sedentárias e praticantes de musculação
}

\section{Low back pain in sedentary women and active}

\author{
Amanda Benvenutti, Altair Argentino Pereira Junior ${ }^{2}$ \\ 'Centro Universitário de Brusque (Unifebe). Brusque, SC, Brasil. \\ ¿Universidade do Estado de Santa Catarina, Florianópolis, SC, Brasil.
}

Recebido em: 28/08/2016 / Aceito em: 10/09/2016 / Publicado em: 18/10/2016

amandabenvenutti@hotmail.com

\section{RESUMO}

Objetivo: identificar a presença de dor lombar em mulheres sedentárias e praticantes de musculação. Método: utilizou-se o questionário de Oswestry, para avaliação da dor lombar. Participaram vinte mulheres com faixa etária entre 50 e 60 anos, divididas em dois grupos. Resultados: Observou-se que as dores na região lombar acabam limitando parte das mulheres, na sua maioria sedentárias, até mesmo de ter uma vida sexual e social normal, além de limitá-las de locomover-se por longas distâncias ou ficar sentadas por um grande período de tempo. O grupo ativo mostrou sentir menor percentual de dor, ao realizar atividade do cotidiano. Considerações finais: no presente estudo, recomenda que a prática da musculação seja algo rotineiro na vida das mulheres, não apenas na faixa etária estudada, mas principalmente que, desde jovem, a mulher exercite-se com cargas, prevenindo um climatério com dores e desconfortos.

Palavras-chave: Treinamento de Resistência; Sedentarismo; Mulheres; Lombalgia.

\section{ABSTRACT}

Objective: to identify the presence of low back pain in women sedentary and bodybuilders. Method: we used the Oswestry questionnaire for evaluation of low back pain. Subjects were twenty women aged between 50 and 60 years, divided into two groups. Results: we observed that low back pain limits several activities of women as, for example, having a sexual and normal social life, and limit them to move around by long distances or remain seated for a long period. The active group showed smaller pain percentage when performing everyday activities. Closing remarks: we recommended that the practice of bodybuilding is something routine in the lives of women, not only in the age group studied, preventing a climacteric with aches and pains.

Keywords: Resistance Training; Sedentary Lifestyle; Women; Low Back Pain.

\section{INTRODUÇÃO}

A dor lombar (DL) é uma situação comum nas sociedades industrializadas, constituindo-se em problema de saúde pública e incapacidade, principalmente para a população economicamente ativa. ${ }^{1}$ Evidências de qualidade moderada apresentaram que, em qualquer região do Brasil, um em cada quatro brasileiros mais velhos sofrem de dor lombar. ${ }^{2}$

Por mais comum que seja a lombalgia, poucos estudos analisam a sua associação com fatores relacionados ao estilo de vida do indivíduo. O que mais encontramos são pesquisas relacionadas à sua prevalência, etiologia e formas de tratamento. ${ }^{3}$

A busca por um estilo de vida mais saudável, pela boa forma física ou até mesmo por um momento de relaxamento fora do stress do trabalho, está fazendo com que, cada dia mais, as salas de musculação se encham de alunos das mais variadas faixas etárias, com os mais variados objetivos e cada um com suas limitações, suas particularidades. Segundo Oliva, Bankoff e Zamai, ${ }^{4}$ é comum encontrar nas academias, pessoas com lesões musculares/articulares causadas pela sobrecarga na musculação. Os autores ainda ressaltam que, embora a prática da musculação seja algo comum na sociedade 
atual, devemos estar alertas com a execução dos exercícios que, na sua maioria, podem causar um aumento da sobrecarga na coluna lombar - exercícios estes que, se realizados de maneira errônea, podem vir a trazer dores nesta região e até graves patologias, como uma hérnia discal, por exemplo.

De acordo com Lopez et al., ${ }^{5}$ pessoas do sexo feminino tem um maior declínio funcional com o passar dos anos, relacionado à perda de massa muscular. A presença de mulheres acima de 50 anos nas academias, buscando a prática da musculação, é cada dia mais comum. Na maioria dos casos, por indicação médica, sabe-se que o exercício resistido, quando bem elaborado e realizado corretamente, pode trazer uma série de benefícios para o organismo de quem o pratica. ${ }^{6}$

Sabe-se que músculos paravertebrais e abdominais fracos, como também a baixa flexibilidade dos músculos da região lombar e posterior da coxa contribuem para o aparecimento de dores na coluna lombar. Quando a lombalgia já está instalada, a prática de exercícios direcionados tem apresentado bons resultados, quanto à diminuição da intensidade da dor, bem como na melhora da funcionalidade. ${ }^{7}$ Estes exercícios podem trazer grandes benefícios se forem executados de maneira correta, com a orientação de um profissional qualificado e manipulando as cargas de acordo com a condição física do indivíduo.

A literatura nos apresenta evidências de que grupos ativos têm menor probabilidade de lesões e dores na coluna lombar, se comparados à grupos sedentários. ${ }^{8}$

No entanto, a postura corporal do praticante principalmente de musculação - é algo que deve ser avaliada constantemente. Por ser uma modalidade onde se trabalha com sobrecargas, uma postura incorreta, durante a realização dos exercícios, pode trazer graves consequências para a coluna lombar. Para Watanabe et al., ${ }^{9}$ os músculos do tronco podem não ser capazes de funcionar adequadamente quando os indivíduos levantam um objeto que é muito mais pesado do que o esperado para seu corpo.

O presente estudo teve como objetivo analisar a presença de dor lombar, em mulheres com faixa etária de 50 a 60 anos, sedentárias e praticantes de musculação.

\section{MÉTODO}

Foram avaliadas vinte participantes, do sexo feminino, com idade entre cinquenta e sessenta anos, divididas em dois grupos: Grupo A - dez mulheres sedentárias, com idade média de 53,4 43,2 anos. As mulheres sedentárias, selecionadas neste estudo, deveriam estar afastadas da prática de atividades físicas regulares por no mínimo um ano. Grupo B - dez mulheres, idade média de 55,9 $9 \pm 4,1$ anos, praticantes regulares de musculação, com uma média de frequência de 3 vezes na semana, por um período mínimo de seis meses. Em ambos os grupos, foram determinados, como critério de exclusão, mulheres que apresentassem diagnóstico clínico de problemas lombares como: histórico de dor lombar crônica, hérnias de disco ou degeneração discal.

Foram selecionadas mulheres praticantes, apenas de musculação. Aquelas que citaram participar de outras atividades conjugadas com o exercício resistido como Zumba, Pilates, dança de salão e natação foram excluídas da amostra para que os resultados não fossem relacionados com nenhum outro tipo de atividade.

As avaliadas, moradoras da cidade de Brusque - Santa Catarina, assinaram um termo de consentimento livre e esclarecido com informações a respeito do artigo a ser realizado. As mesmas responderam ao questionário "Oswestry para avaliação da dor lombar", validado no Brasil, que consta de dez perguntas objetivas a respeito de atividades do cotidiano relacionando com possíveis dores na região lombar. As questões se apresentavam com determinados temas: intensidade da dor no momento da pesquisa; cuidados pessoais; levantar objetos; caminhar; sentar; ficar de pé; dormir; vida sexual; vida social e locomoção em carros/ônibus/taxi.

Após a coleta de dados, estes os resultados foram analisados através de estatística descritiva.

\section{RESULTADOS E DISCUSSÃO}

A tabela 1 apresenta os valores obtidos através da aplicação do questionário de Oswestry, nos dois grupos de participantes e também no total das participantes.

$\mathrm{O}$ estudo identificou que, no momento da pesquisa, a maior parte das entrevistas, tanto do grupo A - Sedentários, quanto do grupo B- Musculação, não apresentava dor lombar. Apenas duas participantes de cada grupo estavam com uma leve dor, outras duas do Grupo A com dor moderada e duas apresentavam dor razoavelmente intensa no momento em que respondiam ao questionário. Para Carey e Freburger, ${ }^{8}$ nos dias atuais, a dor lombar é uma condição comum na população de todo o mundo. Os autores afirmam que $80 \%$ das pessoas apresentarão, em algum momento da vida, um episódio de dor nesta região.

Em dados adicionais, claramente pode-se observar que, um dos itens que mais teve divergência entre os grupos foi o que considerava os cuidados pessoais. Toda a amostra do Grupo B "cuida de si mesmo sem que a dor lombar aumente". Já, no Grupo A, 20\% das entrevistadas "cuida de si mesma, mas sente muita dor ao fazer isso"; e 10\% consegue cuidar de si mesma, porém muito lentamente devido à dor. Normalmente,

Tabela 1 - Valores obtidos por meio do questionário Oswestry no grupo de sedentárias, e praticantes de musculação.

\begin{tabular}{|c|c|c|c|c|}
\hline Faixas percentuais & Tipos de classificação & G. SED n (\%) & G. MUS n (\%) & GERAL n (\%) \\
\hline $0-20 \%$ & Incapacidade mínima & $5(50)$ & $9(90)$ & $14(70)$ \\
\hline $21-40 \%$ & Incapacidade moderada & $2(20)$ & $1(10)$ & $3(15)$ \\
\hline $41-60 \%$ & Incapacidade intensa & $3(30)$ & - & $3(15)$ \\
\hline $61-80 \%$ & Aleijado & - & - & - \\
\hline $81-100 \%$ & Inválido & - & - & - \\
\hline
\end{tabular}

Legenda: G. SED: grupo sedentário; G. MUS: grupo musculação. 
as crises de dor lombar iniciam por volta dos 35 anos e se agravam após os 60 anos. A perda de massa óssea inicia por volta dos 50 anos, sendo mais evidente nas mulheres, sendo o exercício um fator importante na prevenção desta condição ${ }^{10}$.

Levantar objetos pareceu ser mais fácil para muIheres praticantes de musculação, quando comparamos às sedentárias. Apenas $40 \%$ das sedentárias entrevistadas consegue levantar objetos pesados sem aumento da dor; $30 \%$ pode levantar objetos pesados, porém isto acentua a dor na lombar; $10 \%$ não consegue levantar objetos pesados por causa da dor, mas objetos leves se estiverem bem posicionados, as participantes relataram conseguir; e $20 \%$ delas consegue levantar apenas objetos muito leves. Das praticantes de musculação, $80 \%$ consegue levantar objetos pesados sem aumento da dor; $10 \%$ levanta objetos pesados apenas se estiverem bem posicionados, por exemplo, sobre uma mesa; e 10\% pode levantar apenas objetos muito leves. O que está de acordo com o estudo de Rocha et al., ${ }^{11}$ que observaram que um programa de exercício físico atenuou o aumento dos níveis de adiposidade e a perda muscular associada à menopausa e ao envelhecimento em mulheres.

Para Carvalho, ${ }^{12}$ a caminhada é uma atividade com baixo risco de lesão que está relacionada à muitos benefícios para a saúde, o que segundo ele, poderia ser promissor para indivíduos com lombalgia. No presente estudo, cem por cento das praticantes de musculação entrevistadas não sente dor na região lombar, ao caminhar qualquer distância. Já, as sedentárias mostraram ter mais dificuldade quando a questão é caminhar longas distâncias: $20 \%$ delas não pode caminhar mais que 1600 metros e $30 \%$ sente dor se caminha mais de 400 metros.

Das dez praticantes de musculação entrevistadas, sete conseguem sentar em qualquer tipo de cadeira, durante o tempo quiser, e as outras três conseguem permanecer sentadas o tempo que quiserem se a cadeira for confortável. Do grupo das sedentárias, quatro afirmaram conseguir permanecer sentada em qualquer tipo de cadeira, durante o tempo que quiserem e outras quatro afirmaram o mesmo, porém precisam de uma cadeira confortável; e duas mulheres não conseguem ficar sentadas por mais de meia hora sem que sintam dor.

A postura sentada repercute em fator de risco para a coluna vertebral, ela a afeta promovendo um desequilíbrio das estruturas de sustentação. Ao manter-se sentado em uma cadeira comum a lordose lombar retifica, enquanto que a porção posterior dos ligamentos tensiona-se, além de propiciar o aumento da pressão sobre os discos intervertebrais lombares. ${ }^{13}$

Para o Grupo A, ficar de pé sem que sinta dor na lombar é possível, apenas para quatro das entrevistadas; três se queixaram de dor ao ficar de pé; outras duas disseram não poder ficar mais de meia hora de pé, pois a dor aumenta e uma mulher afirmou não conseguir ficar de pé por mais de uma hora sem que a dor aumente. Já, para o Grupo B, sete mulheres conseguem ficar de pé o tempo que quiserem sem sentir dor; duas fazem isto, porém com aumento da dor; e apenas uma entrevistada se queixou de não conseguir ficar de pé por mais de uma hora. Uma possível explicação para o aumento da dor na região lombar ao ficar de pé, é a hipotonia da musculatura que fica nesta região, comum em indivíduos sedentários, porém praticantes de musculação que não fazem exercícios específicos para esta musculatura, tendem a ter dor na lombar também.

O exercício físico, como recurso terapêutico para a prevenção e tratamento da dor lombar, tem recebido grande atenção nos últimos anos, o que pode ser explicado pelos consistentes relatos de que a fraqueza e a baixa resistência isométrica dos músculos eretores lombares da coluna estão associadas à etiologia da dor lombar. ${ }^{7,8}$

Apenas duas mulheres das dez entrevistadas no grupo das praticantes de musculação afirmaram ter o sono perturbado ocasionalmente pela dor lombar; as demais não apresentam perturbação no sono por causa da dor. No grupo das sedentárias, uma mulher afirmou ter o sono perturbado ocasionalmente pela dor e duas disseram não conseguir dormir mais de seis horas por causa da dor; as demais do grupo não apresentaram problemas com o sono devido à dor. Acredita-se este ser um tópico bastante relativo ao tipo de colchão e à posição em que a entrevistada dorme, já que teoricamente, a posição deitada é a que menor tensiona a região lombar.

Oliveira, Salgueiro e Alfieri ${ }^{14}$ ressaltam que as posturas inadequadas associadas à repetição e ineficiência - fraqueza/hipotonia - muscular podem desencadear a lombalgia. É como uma cadeia de fatores que se interligam um com o outro: Hipotonia muscular gera vícios posturais maléficos para a saúde da coluna que com o passar do tempo podem levar o indivíduo a sentir dores, no caso na lombar que, em geral, é a região da coluna vertebral que mais sofre com este tipo de conduta.

A vida sexual e social das entrevistadas do Grupo B se mostrou completamente normal, segundo os resultados. Já, no Grupo A, $10 \%$ afirmou ter vida sexual normal, porém com um pouco de dor e $20 \%$ com muita dor lombar. Apenas metade das entrevistadas do Grupo A, tem vida social normal sem dor; $10 \%$ tem vida social normal, porém com aumento da dor e 40\% tem vida social com limitações quando se trata de interesses que demandam mais energia.

Em um estudo sobre a intervenção fisioterapêutica no impacto da dor lombar crônica em idosos, Jorge et $\mathrm{al}^{15}$, citam a dor lombar como uma das principais queixas entre os idosos. Os autores afirmam ainda que a dor está entre os principais fatores que limitam a possibilidade do idoso em manter seu cotidiano de maneira normal, prejudicando, de algum modo, a realização das atividades de vida diária bem como restringindo a convivência social.

Quando o assunto é locomoção (carro, ônibus, taxi), o Grupo $A$ apresentou mais queixas em relação a dores na região lombar: três mulheres afirmaram ir a qualquer lugar, porém com aumento da dor e uma entrevistada disse ter dor intensa, conseguindo locomover-se por duas horas. No grupo B apenas uma mulher disse que pode ir a qualquer lugar, mas isso aumenta a dor. Neste tópico, se aplica o mesmo princípio utilizado na questão que trata de ficar na postura sentada por um longo período de tempo. A compressão discal aumenta a tensão sobre os ligamentos da coluna lombar também, gerando um aumento da dor.

Segundo a escala "Oswestry para avaliação da dor lombar", das 20 mulheres entrevistadas, 14 (70\%) 
apresentam incapacidade mínima. Destas 14, 9 são praticantes de musculação e as outras 5 são sedentárias. No total, 3 mulheres (15\%) apresentaram incapacidade moderada, sendo 2 sedentárias e 1 praticante de musculação. E, para incapacidade intensa, obteve-se 3 entrevistadas (15\%), todas sedentárias.

Sabe-se que a inatividade física está relacionada direta ou indiretamente com dores na coluna. A hipotonia da musculatura lombar - presente em pessoas sedentárias - associada com postura inadequada é uma combinação perfeita para o surgimento da lombalgia.

Comparando os escores entre os grupos, pode-se ver que o grupo sedentário apresenta maior incapacidade em relação ao grupo praticante de musculação. Vale ressaltar a partir daí, a importância da prática de musculação para mulheres, principalmente na faixa etária estudada, fase do climatério - menopausa. Como já falado anteriormente, o fortalecimento da musculatura do corpo por um todo, auxilia na prevenção de dores, principalmente, na região lombar, que é comum ser afetada devido à sobrecarga que recebe diariamente. Jesus e Marinho ${ }^{16}$ e Albino et al., ${ }^{17}$ afirmaram que o exercício físico ajuda a amenizar os principais fatores envolvidos na "síndrome da dor lombar". Segundo os autores, dentre estes fatores estão a fraqueza muscular - da região abdominal, principalmente, e a baixa flexibilidade dorsal e de membros inferiores.

\section{CONSIDERAC̣ÕES FINAIS}

Ao comparar as atividades da vida diária de mulheres sedentárias, com mulheres que praticam exercícios com cargas, nota-se nitidamente que as ativas conseguem realizar suas necessidades do cotidiano - caminhar, sentar, vestir-se, viajar, vida sexual, cuidados pessoais e socialização - com ausência, ou muito pouca dor na região lombar. Já, as sedentárias, mostraram-se ter uma maior restrição com relação às atividades diárias, pois a dor as limita de fazer parte das atividades e situações do cotidiano.

Com este estudo, pode-se ressaltar a importância da musculação para mulheres, principalmente, na faixa etária estudada - dos 50 aos 60 anos - que é uma fase crítica para a mulher, com a chegada da menopausa e com ela uma série de dores relacionadas à desgastes ósseos e perda de massa muscular.

O ideal seria que as mulheres nunca parassem de praticar exercícios físicos. Seria interessante que toda mulher praticasse algum exercício durante toda a vida não necessariamente só musculação, mas principalmente ela, por ser uma das modalidades que mais traz benefícios, quando se fala de fortalecimento muscular e prevenção de lesões/dores provindas com a idade. Assim, quando chegada a fase do climatério, o corpo já se encontra 'preparado' para as mudanças - com músculos e ossos fortes, e consequentemente, articulações protegidas.

As mulheres sedentárias devem ter cuidados com a postura adotada no dia-a-dia, para prevenir episódios de dor lombar, no entanto, o ideal seria sair desse estado de sedentarismo e buscar atividades físicas que promovam fortalecimento dos grupos musculares, evitando assim, episódios de dor lombar. Já, as mulheres praticantes de musculação, devem manter seus treinos, objetivando a manutenção do seu estado de saúde.

\section{REFERÊNCIAS}

1. Meziat-Filho N, Silva GA. Disability pension from back pain among social security beneficiaries, Brazil. Rev Saude Publica 2011;45(3):494-502. doi: 10.1590/S003489102011000300007

2. Leopoldino AAO, Diz JBM, Martins VT, Henschke N, Pereira LSM, Dias RC, Oliveira VC. Prevalence of low back pain in older Brazilians: a systematic review with meta-analysis. Rev Bras Reumatol 2016;56(3):258-269. doi: 10.1016/j. rbre.2016.03.011.

3. Hoy D, March L, Brooks P, Blyth F, Woolf A, Bain C, Williams G, Smith E, Vos T, Barendreget J, Murray C, Burstein R, Buchbinder R. The global burden of low back pain: estimates from the Global Burden of Disease 2010 study. Ann Rheum Dis 2014;73(6):968-74. doi: 10.1136/ annrheumdis-2013-204428.

4. Oliva OJ, Bankoff ADP, Zamai CA. Possíveis lesões musculares e ou articulares causadas por sobrecarga na prática da musculação. RBAFS, Campinas 1998;3(3):15-23.

5. Lopez P, Radaelli R, Rech A, Wilhelm EN, Pinto RS. Muscle quality, but not muscle thickness, is decreased in different age groups of active older women. Rev bras cineantropom desempenho hum 2015;17(3):347-356. doi: 10.5007/1980-0037.2015v17n3p347.

6. Lessa P, Oshita TAD, Valezzi M. Quando as mulheres invadem as salas de musculação: aspectos biossociais da musculação e da nutrição para mulheres. Iniciação Científica Cesumar 2007;9(2):109-117.

7. Soares P, Cabral V, Mendes M, Vieira R, Avolio G, Vale RGS. Efeitos do Programa Escola de Postura e Reeducação Postural Global sobre a amplitude de movimento e níveis de dor em pacientes com lombalgia crónica. Rev Andal Med Deporte 2016;9(1):23-28. doi: 10.1016/j.ramd.2015.02.005.

8. Carey TS, Freburger JK. Exercise and the Prevention of Low Back Pain. JAMA Intern Med 2016;176(2):208-209. doi: 10.1001/jamainternmed.2015.7636.

9. Watanabe M, Kaneoka K, Okubo Y, Shiina I, Tatsumura M, Miyakawa S. Trunk muscle activity while lifting objects of unexpected weight. Physiotherapy 2013;99(1):78-83. doi: 10.1016/j.physio.2011.09.005.

10. Liu BX, Chen SP, Li YD, Wang J, Zhang B, Lin Y, Guan JH, Cai YF, Liang Z, Zheng F. et al. The effect of the modified eighth section of eight-section brocade on osteoporosis in postmenopausal woman: A prospective randomized trial. Medicine (Blatimore) 2015;94(25):e991. doi: 10.1097/MD.0000000000000991.

11. Rocha SBR, Ogando BMA, Reis VMCP, Ávila WRM, Carneiro AG, Gabriel RECD, Moreira MHR. Impacto de um programa de exercício físico na adiposidade e na condição muscular de mulheres pós-menopáusicas. Rev Bras Ginecol Obstet 2012;34(9):414-419. doi: http://dx.doi.org/10.1590/ S0100-72032012000900005.

12. Carvalho AR. Alterações nas variáveis mecânicas e energéticas da caminhada decorrentes da dor lombar crônica inespecífica: estudo observacional transversal. 107 f. [Dissertação]. Universidade Federal do Rio Grande do Sul, Porto Alegre, 2012.

13. Estupiñan ZTG, García MFM, Correal FE. Low back pain perception from the prolonged use of a dynamic seat in sitting posture. Rev Salud Pública 2016;18(3):412-424. doi: 10.15446/rsap.v18n3.42897. 
14. Oliveira JG, Salgueiro MMHAO, Alfiere FM. Lombalgia e estilo de vida. UNOPAR Científica Ciências Biológicas e da Saúde. São Paulo 2014;16(4):341-344.

15. Jorge MSG, Zanin C, Knob B, Wibelinger. Physiotherapeutic intervention on chronic lumbar pain impact in the elderly. Rev Dor 2015;16(4):302-5. doi: 10.5935/1806-0013.20150062 .

16. Jesus GT, Marinho ISF. Causas de lombalgia em grupos de pessoas sedentárias e praticantes de atividades físicas. Ano 10, n. 92, Efdeportes, 2006. Disponível em: http://www. efdeportes.com/efd92/lombal.htm Acesso: 10 jul 2016.

17. Albino NT. Pilates e lombalgia: efetividade do transverso abdominal, capacidade funcional e qualidade de vida. Fisioterapia Brasil 2011;12(4):273-278.

Como citar: PEREIRA JÚNIOR, Altair Argentino; BENVENUTTI, Amanda. Dor Lombar em Mulheres Sedentárias e Praticantes de Musculação. Cinergis, Santa Cruz do Sul, v. 18, n. 1, out. 2016. ISSN 2177-4005. Disponível em: <https://online. unisc.br/seer/index.php/cinergis/article/view/7903>. Acesso em: 11 out. 2016. doi:http://dx.doi.org/10.17058/cinergis v18i1.7903. 